\title{
Rank M-type Filters for Image Denoising
}

\author{
Francisco J. Gallegos-Funes and Alberto J. Rosales-Silva \\ National Polytechnic Institute of Mexico \\ Mexico
}

\section{Introduction}

Many different classes of filters have been proposed for removing noise from images (Astola \& Kuosmanen, 1997; Bovik, 2000; Kotropoulos \& Pitas, 2001). They are classified into several categories depending on specific applications. Linear filters are efficient for Gaussian noise removal but often distort edges and have poor performance against impulsive noise. Nonlinear filters are designed to suppress noise of different nature, they can remove impulsive noise and guarantee detail preservation. Rank order based filters have received considerable attention due to their inherent outlier rejection and detail preservation properties.

In the last decade, many useful techniques of multichannel signal processing based on vector processing have been investigated due to the inherent correlation that exists between the image channels compared to traditional component-wise approaches. Many applications of this technique are color image processing, remote sensing, robot vision, biomedical image processing, and high-definition television (HDTV). Different filtering techniques have been proposed for color imaging (Plataniotis \& Venetsanopoulos, 2000). Particularly, nonlinear filters applied to color images have been designed to preserve edges and details, and remove impulsive noise. On the other hand, the filters based in the wavelet domain provide a better performance in terms of noise suppression in comparison with different spatial domain filters (Mahbubur Rahman \& Kamrul Hasan, 2003).

The possibility to process $3 \mathrm{D}$ images presents a new application where it is necessary to improve the quality of 3D objects inside the image, suppressing a noise of different nature (impulsive, Gaussian noise, or may be by speckle one) that always affects the communication or acquisition process (Nikolaidis \& Pitas, 2001). Multiplicative (speckle) noise is common for any system using a coherent sensor, for example, the ultrasound transducer. Other problem that is not trivial is the adaptation and implementation of the current filters, that have been investigated in different papers in the case of $2 \mathrm{D}$ image processing to process objects in $3 \mathrm{D}$ by use multiframe methods to increase the signal-tonoise ratio (SNR).

This chapter presents the capability features of robust Rank M-Type K-Nearest Neighbor (RMKNN) and Median M-Type L- (MML) filters for the removal of impulsive noise in grayscale image processing applications (Gallegos \& Ponomaryov, 2004; Gallegos-Funes et al., 2005; Gallegos-Funes et al., 2008). The proposed scheme is based on combined robust $R-$ (median, Wilcoxon, Ansari-Bradley-Siegel-Tukey or Mood) and M-estimators, and modification of the KNN and L- filters that use the RM (Rank M-type) -estimator to calculate 
the robust point estimate of the pixels within the filtering window. So, such filters use the value of the central pixel within the filtering window to provide the preservation of fine details and the redescending $M$-estimators combined with the calculation of the rank estimator to obtain the sufficient impulsive noise rejection. Different types of influence functions in the $M$-estimator can be used to provide better impulsive noise suppression. We apply the proposed MML filter in SAR images which naturally have speckle noise to demonstrate that the speckle noise can be efficiently suppressed, while the sharpness and fine feature are preserved.

The robust Rank M-Type K-Nearest Neighbor (RMKNN) filters are adapted to work in color image denoising (Ponomaryov et al., 2005). We also present the 3D RMKNN and 3D MML filters which are compared with different nonlinear 2D filters which were adapted to $3 \mathrm{D}$ (Varela-Benitez et al., 2007a). The experimental results were realized by degraded an ultrasound sequence with different variance of speckle noise added to the natural speckle noise of the sequence. Finally, we adapt the RMKNN, MML, and different order statistics filters to work in the wavelet domain for the removal of impulsive and speckle noise in gray-scale and color image processing applications (Gallegos-Funes et al., 2007; VarelaBenitez et al., 2007b).

Another goal of this chapter is to demonstrate the possibility to implement the filters in order to process the image or sequence in real time by means of use of the Texas Instruments DSP TMS320C6701 and DSP TMS320C6711 to demonstrate that the proposed methods potentially could provide a real-time solution to quality video transmission.

Extensive simulation results with different gray scale and color images and video sequences have demonstrated that the proposed filters consistently outperform other filters by balancing the tradeoff between noise suppression, fine detail preservation, and color retention.

\section{Rank M-type estimators}

\subsection{R-estimators}

The $R$-estimators are a class of nonparametric robust estimators based on rank calculations (Hampel et al., 1986; Huber, 1981). We consider a two-samples of rank tests $x_{1}, \ldots, x_{m}$ and $y_{1}, \ldots, y_{n}$ as a two-samples with distributions $H(x)$ and $H(x+\Delta)$, where $\Delta$ is the shift of unknown location. Let $X_{(i)}$ be the rank of $X_{i}$ in the pooled sample of size $N=m+n$. The rank test of $\Delta=0$ against $\Delta>0$ is based on the statistics test,

$$
S=\frac{1}{m} \sum_{i=1}^{m} a_{i}\left(X_{(i)}\right)
$$

Usually, the scores $a_{i}$ are generated by the function $J$ as follows:

$$
a_{i}=N \int_{(i-1) / N}^{i / N} J(t) d t
$$

The function $J(t)$ is symmetric in the sense of $J(1-t)=-J(t)$, satisfies $\int J(t) d t=0$ and the coefficients $a_{i}$ are given as $\sum_{i=1}^{n} a_{i}=0$. 
The median estimator can be derived from Laplace distribution function $f_{0}(x)=\frac{1}{2} e^{-|x|}$ with function $J(t)=\left\{\begin{array}{ll}-1 & t<\frac{1}{2} \\ 1 & t>\frac{1}{2}\end{array}\right.$ and is given in such a form (Hampel et al., 1986; Huber, 1981):

$$
\hat{\theta}_{\text {med }}= \begin{cases}\frac{1}{2}\left(X_{(n / 2)}+X_{(1+n / 2)}\right), & \text { for even } n \\ X_{\left(\frac{n+1}{2}\right)}, & \text { for odd } n\end{cases}
$$

where $X_{(j)}$ is the element with rank $j$. It is the best estimator when any a priori information about data $X_{i}$ distribution shape and its moments is unavailable.

The Hodges-Lehmann estimator $J(t)=\left|t-\frac{1}{2}\right|$ is relational with Wilcoxon test and logistic distribution function $f_{0}(x)=\frac{1}{1+e^{-x}}$. The corresponding rank estimator is the Wilcoxon $R$ estimator (Hampel et al., 1986; Huber, 1981):

$$
\hat{\theta}_{\text {Wil }}=\operatorname{MED}_{i \leq j}\left\{\frac{1}{2}\left(X_{(i)}+X_{(j)}\right), i, j=1, \ldots N\right\}
$$

The Wilcoxon estimator is robust and unbiased. When the shape of the original data distribution is symmetrical, the such a test is known as the local asymptotically most powerful one.

Other R-estimations can be obtained by different type of functions $J(t)$. For example, the functions Ansari-Bradley-Siegel-Tukey $J(t)=\left|t-\frac{1}{2}\right|-\frac{1}{4} \quad$ and Mood $J(t)=\left(t-\frac{1}{2}\right)^{2}-\frac{1}{12}$ determine the R-estimators (Hampel et al., 1986; Huber, 1981):

$$
\begin{gathered}
\hat{\theta}_{\mathrm{ABST}}=\operatorname{MED}\left\{\begin{array}{ll}
X_{(i)}, & i \leq\left[\frac{N}{2}\right] \\
\frac{1}{2}\left(X_{(i)}+X_{(j)}\right), & {\left[\frac{N}{2}\right]<i \leq N}
\end{array}\right\} \\
\hat{\theta}_{\mathrm{MOOD}}=\operatorname{MED}\left\{\begin{array}{ll}
\frac{1}{2}\left(X_{(i)}+X_{(j)}\right), & i \leq 3 \\
X_{(i)}, & 3<i \leq N
\end{array}\right\}
\end{gathered}
$$

\subsection{M-estimators}

The $M$-estimators are a generalization of maximum likelihood estimators (MLE) (Hampel et al., 1986; Huber, 1981). Their definition is given by a function $\rho\{\rho(X)=\ln (F(X))\}$ connected with the probability density function $F(X)$ of data samples $X_{i}, i=1, \ldots, N$ : 


$$
\hat{\theta}=\underset{\theta \in \Theta}{\arg \min } \sum_{i=1}^{N} \rho\left(X_{i}-\theta\right)
$$

The estimation of the location parameter $\theta$ can be found by calculating the partial derivative of $\rho$ (with respect to $\theta$ ) introducing the function $\psi(X, \theta)=\frac{\partial}{\partial \theta} \rho(X, \theta)$

$$
\sum_{i=1}^{N} \psi\left(X_{i}-\theta\right)=0
$$

where $\theta$ is a location parameter.

The robust $M$-estimator solution for $\theta$ is determined by imposing certain restrictions on the influence function $\psi(X)$ or the samples $X_{i}-\theta$, called censorization or trimming. The standard technique for the $M$-estimator assumes the use of Newton's iterative method that can be simplified by a single-step algorithm to calculate the lowered $M$-estimate of the average $\theta$ value (Astola \& Kuosmanen, 1997)

$$
\theta_{\mathbf{M}}=\frac{\sum_{i=1}^{N} X_{i} \widetilde{\psi}\left(X_{i}-\operatorname{MED}\{\vec{X}\}\right)}{\sum_{i=1}^{N} 1_{[-r . r]}\left(X_{i}-\operatorname{MED}\{\vec{X}\}\right)}
$$

where $\widetilde{\psi}$ is the normalized function $\psi: \psi(X)=X \widetilde{\psi}(X)$. It is evident that (9) represents the arithmetic average of $\sum_{i=1}^{n} \psi\left(X_{i}-\operatorname{MED}\{\overrightarrow{\mathbf{X}}\}\right)$, which is evaluated on the interval $[-r, r]$. The parameter $r$ is connected with restrictions on the range of $\psi(X)$, for example, as it has been done in case of the simplest Huber's limiter type $M$-estimator for the normal distribution having heavy 'tails' (Huber, 1981)

$$
\widetilde{\psi}_{r}(X)=\min (r, \max (X, r))=[X]_{-r}^{r}
$$

Another way to derive the function $\widetilde{\psi}(X)$ is to cut the outliers off the primary sample. This leads to the so-called lowered $M$-estimates. Hampel proved that the skipped median is the most robust lowered $M$-estimate (Hampel et al., 1986). Below we also use the simple cut (S) function. There exist also other well known influence functions in the literature. We also use the Hampel's three part redescending $(\mathrm{H})$, Andrew's sine $(\mathrm{A})$, Tukey biweight $(\mathrm{T})$, and the Bernoulli (B) influence functions (Astola \& Kuosmanen, 1997; Hampel et al., 1986). These functions are shown in Table 1.

\subsection{RM-estimators}

The proposal to enhance the robust properties of $M$-estimators and $R$-estimators by using the $R$-estimates consists of the procedure similar to the median average instead of arithmetic one (Gallegos-Funes et al., 2002; Gallegos \& Ponomaryov 2004): 


$$
\begin{gathered}
\theta_{\text {medM }}=\operatorname{MED}\left\{X_{i} \widetilde{\psi}\left(X_{i}-\operatorname{MED}\{\overrightarrow{\mathbf{X}}\}\right), i=1, \ldots, N\right\} \\
\theta_{\text {WilM }}=\operatorname{MED}_{i \leq j}\left\{\frac{1}{2}\left[X_{i} \widetilde{\psi}\left(X_{i}-\operatorname{MED}\{\overrightarrow{\mathbf{X}}\}\right)+X_{j} \widetilde{\psi}\left(X_{j}-\operatorname{MED}\{\overrightarrow{\mathbf{X}}\}\right)\right], i=1, \ldots, N\right\}
\end{gathered}
$$

\begin{tabular}{|c|c|}
\hline Influence function & Formulae \\
\hline Simple cut (S) & $\psi_{\operatorname{cut}(r)}(X)=X \cdot 1_{[-r, r]}(X)=\left\{\begin{array}{l}X,|X| \leq r \\
0,|X|>r\end{array}\right.$ \\
\hline $\begin{array}{c}\text { Hampel's three part } \\
\text { redescending (H) }\end{array}$ & $\psi_{\alpha, \beta, r}(X)=\left\{\begin{array}{c}X, 0 \leq|X| \leq \alpha \\
\alpha \cdot \operatorname{sgn}(X), \quad \alpha \leq|X| \leq \beta \\
r-|X| \\
\alpha \frac{r-\beta}{r}, \beta \leq|X| \leq r \\
0, r \leq|X|\end{array}\right.$, where $0<\alpha<\beta<r<\infty$. \\
\hline Andrew's sine (A) & \begin{tabular}{c}
$\psi_{\sin (r)}(X)=\left\{\begin{array}{c}\sin (X / r),|X| \leq r \pi \\
0,|X|>r \pi\end{array}\right.$ \\
\hline Tukey's biweight (T)
\end{tabular} \\
\hline Bernoulli (B) & $\psi_{\operatorname{bi}(r)}(X)=\left\{\begin{array}{c}X^{2}\left(r^{2}-X^{2}\right),|X| \leq r \\
0,|X|>r\end{array}\right.$ \\
\hline$\psi_{\operatorname{ber}(r)}(X)=X^{2} \sqrt{r^{2}-X^{2} \cdot 1_{[-r, r]}(X)}$ \\
\hline
\end{tabular}

Table 1. Influence functions used in the filtering scheme to derive the robust redescending M-estimators.

Such an estimator is the combined RM-estimator. It should be noted that the RM-estimator (11) is the usual median when the function $\tilde{\psi}$ is represented by eq. (10). If the function $\tilde{\psi}$ is described by the simple cut function, it yields the skipped median. Other new RMestimators applied below are followed from eqs. (5) and (6) (Gallegos-Funes et al., 2005):

$$
\begin{aligned}
& \theta_{\text {AвSTM }}=\operatorname{MED}_{i \leq j}\left\{\begin{array}{ll}
X_{i} \widetilde{\psi}\left(X_{i}-\operatorname{MED}\{\overrightarrow{\mathbf{X}}\}\right), & i \leq \frac{N}{2} \\
\frac{1}{2}\left[X_{i} \widetilde{\psi}\left(X_{i}-\operatorname{MED}\{\overrightarrow{\mathbf{X}}\}\right)+X_{j} \widetilde{\psi}\left(X_{j}-\operatorname{MED}\{\overrightarrow{\mathbf{X}}\}\right)\right], & \frac{N}{2}<i \leq N
\end{array}\right\} \\
& \theta_{\mathrm{MoodM}}=\operatorname{MED}_{i \leq j}\left\{\begin{array}{ll}
\frac{1}{2}\left[X_{i} \widetilde{\psi}\left(X_{i}-\operatorname{MED}\{\overrightarrow{\mathbf{X}}\}\right)+X_{j} \widetilde{\psi}\left(X_{j}-\operatorname{MED}\{\overrightarrow{\mathbf{X}}\}\right)\right], & i \leq 3 \\
X_{i} \widetilde{\psi}\left(X_{i}-\operatorname{MED}\{\overrightarrow{\mathbf{X}}\}\right), & 3<i \leq N
\end{array}\right\}
\end{aligned}
$$

It is possible to expect that the robust properties of the RM-estimators can exceed the robust properties of the base $R$ - and $M$ - estimators. The $R$-estimator provides good properties of impulsive noise suppression and the $M$-estimator uses different influence functions according to the scheme proposed by Huber to provide better robustness, for these reasons it can be expected that the properties of combined RM-estimators could be better in 
comparison with $R$ - and $M$ - estimators (Gallegos-Funes et al., 2002; Gallegos \& Ponomaryov 2004).

\section{Rank M-type filters}

To increase the robustness of standard filters, it is possible to employ different methods known in the robust-estimate theory, for example, the censoring or others (Astola \& Kuosmanen, 1997; Peltonen \& Kuosmanen, 2001). The known proposal to increase the quality of filtration via the preservation both the edges and small-size details in the image consists of the use of KNN image-filtering algorithm. Other proposal filtering schemes proposed here are the L-filter and the versions of KNN and L filters in wavelet domain.

\subsection{Rank M-Type KNN filters}

The following representation of the KNN filter is usually used $\theta_{\mathrm{KNN}}=\sum_{i=1}^{n} a_{i} x_{i} / \sum_{i=1}^{n} a_{i}$ with $a_{i}=\left\{\begin{array}{cc}1, & \text { if }\left|x_{i}-x_{c}\right| \leq T \\ 0, & \text { otherwise }\end{array}, x_{c}\right.$ is the central pixel, and $T$ is a threshold (Astola \& Kuosmanen, 1997). If the threshold $T$ is chosen to be twice of the standard noise deviation $\sigma$, this filter is known as the sigma filter. The KNN filter can be rewritten as $\hat{\theta}_{\mathrm{KNN}}(i, j)=\frac{1}{K} \sum_{m=-L}^{L} \sum_{n=-L}^{L} \psi(x(i+m, j+n)) x(i+m, j+n) \quad$ where $\quad x(i+m, j+n) \quad$ are the pixels in the filter window, $m, n=-L, \ldots, L$, and $\psi(x(i+m, j+n))=\left\{\begin{array}{ll}1, & \text { if } x(i+m, j+n) \text { are } K \text { sampleswhose valuesareclosest to } \\ \text { the valueof the centralsample } x \text { inside the filter window } & \text { otherwise }\end{array}\right.$.

To improve the robustness of the KNN filter that can preserve well both the edges and the fine details in absence of noise, we proposed to use the RM-estimator (11) given by $\hat{\theta}_{\text {MMKNN }}(i, j)=\operatorname{MED}\left\{\hat{\theta}_{\mathrm{KNN}}(i+m, j+n)\right\}$. So, the iterative Median M-type K-Nearest Neighbor filter can be written as (Gallegos-Funes et al., 2002; Gallegos \& Ponomaryov 2004),

$$
\theta_{\text {MMKNN }}^{(w)}(i, j)=\operatorname{MED}\left\{h^{(w)}(i+m, j+n)\right\}
$$

where $h^{(w)}(i+m, j+n)$ is a set of $K_{\text {close }}$ values of pixels weighting in accordance with the used $\widetilde{\psi}(X)$ influence function within the filter window closest to the estimate obtained at previous step $\hat{\theta}_{\text {MMKNN }}^{(w-1)}(i, j)$. The initial estimate is $\hat{\theta}_{\text {MMKNN }}^{(0)}(i, j)=x(i, j)$ and $\hat{\theta}_{\text {MMKNN }}^{(w)}(i, j)$ denotes the estimate at the iteration $w . x(i, j)$ is the current or origin pixel contaminated by noise in the filtering window. The filtering window size is $N=(2 L+1)^{2}$ and $m, n=-L, \ldots, L$. The current number of the nearest neighbor pixels $K_{\text {close }}$ reflects the local data activity and impulsive noise presence (Gallegos \& Ponomaryov 2004),

$$
K_{\text {close }}= \begin{cases}K_{\min }+a D_{S}, & \left(K_{\min }+a D_{S}\right) \leq K_{\max } \\ K_{\max }, & \text { otherwise }\end{cases}
$$


The parameter $a$ controls the filter sensitivity for local data variance to detect the details. $K_{\min }$ is the minimal number of neighbours for noise removal and $K_{\max }$ is the maximal number of neighbours for edge restriction and detail smoothing. $D_{S}$ is the impulsive detector, and MAD is the median of the absolute deviations from the median (Astola \& Kuosmanen, 1997) which are defined as (Gallegos \& Ponomaryov 2004):

$$
\begin{gathered}
D_{S}=\frac{\operatorname{MED}\{|x(i, j)-x(i+m, j+n)|\}}{\operatorname{MAD}\{x(i, j)\}}+0.5 \frac{\operatorname{MAD}\{x(i, j)\}}{\operatorname{MED}\{x(i+k, j+l)\}} \\
\operatorname{MAD}\{x(i, j)\}=\operatorname{MED}\{\mid x(i+m, j+n)-\operatorname{MED}\{x(i+k, j+l)\}\}\}
\end{gathered}
$$

In our experiments, a $3 \times 3$ window (i.e., $m, n=-1, \ldots, 1$ and $\left.(2 L+1)^{2}=9\right)$ is applied. The algorithm finishes when $\hat{\theta}_{\text {MMKNN }}^{(w)}(i, j)=\hat{\theta}_{\text {MMKNN }}^{(w-1)}(i, j)$. The use of the influence functions mentioned above in the proposed filter (15) could provide good suppression of impulsive noise. We also propose for enhancement of the removal ability of MMKNN filter in the presence of impulsive noise to involve the standard median filter. The numerical simulations have shown that for $K_{\text {close }}>7$ the MMKNN filter can be substituted by the $3 \times 3$ median filter and for $K_{\text {close }}>350$ we can use the $5 \times 5$ median filter.

Other versions of Rank M-type K-Nearest Neighbor filters are given as follows (GallegosFunes et al., 2005; Ponomaryov et al., 2005),

$$
\begin{aligned}
& \hat{\theta}_{\mathrm{WMKNN}}^{(w)}(i, j)=\operatorname{MED}\left\{\frac{h^{(w)}(i+m, j+n)+h^{(w)}\left(i+m_{1}, j+n_{1}\right)}{2}\right\} \\
& \hat{\theta}_{\text {ABSTMKNN }}^{(w)}(i, j)=\operatorname{MED}_{i \leq j}\left\{\begin{array}{ll}
h^{(w)}(i+m \cdot j+n), & i, j \leq \frac{N}{2} \\
\frac{h^{(w)}(i+m, j+n)+h^{(w)}\left(i+m_{1}, j+n_{1}\right)}{2}, & \frac{N}{2}<i
\end{array}\right\} \\
& \hat{\theta}_{\text {MOODMKNN }}^{(w)}(i, j)=\operatorname{MED}_{i \leq j}\left\{\begin{array}{ll}
h^{(w)}(i+m \cdot j+n), & 3<i \leq N \\
\frac{h^{(w)}(i+m, j+n)+h^{(w)}\left(i+m_{1}, j+n_{1}\right)}{2}, & i \leq 3
\end{array}\right\}
\end{aligned}
$$

\subsection{Wavelet domain order statistics filter}

This filter constitutes two filters (Gallegos-Funes et al., 2007): the filter based on redundancy of approaches (Gallegos-Funes et al., 2007) and the Wavelet domain Iterative Center Weighted Median (ICWMF) Filter (Mahbubur Rahman \& Kamrul Hasan, 2003) as shown in Figure 1. For each color component of the noisy image it is necessary to apply all the steps contained in this structure. This technique applies up to 5 scaling levels for the details and only 1 scaling level for the approaches. Other operations are indicated to make clearer the wavelet analysis that it is carried out in this paper. We modify this structure in the block of the ICWMF. For that reason, the expressions used by the ICWMF to calculate the improved estimation of the variance field of the noisy wavelet coefficients will be required to indicate 
when and where different proposed filtering algorithms will take place to improve the performance of the proposed filter.

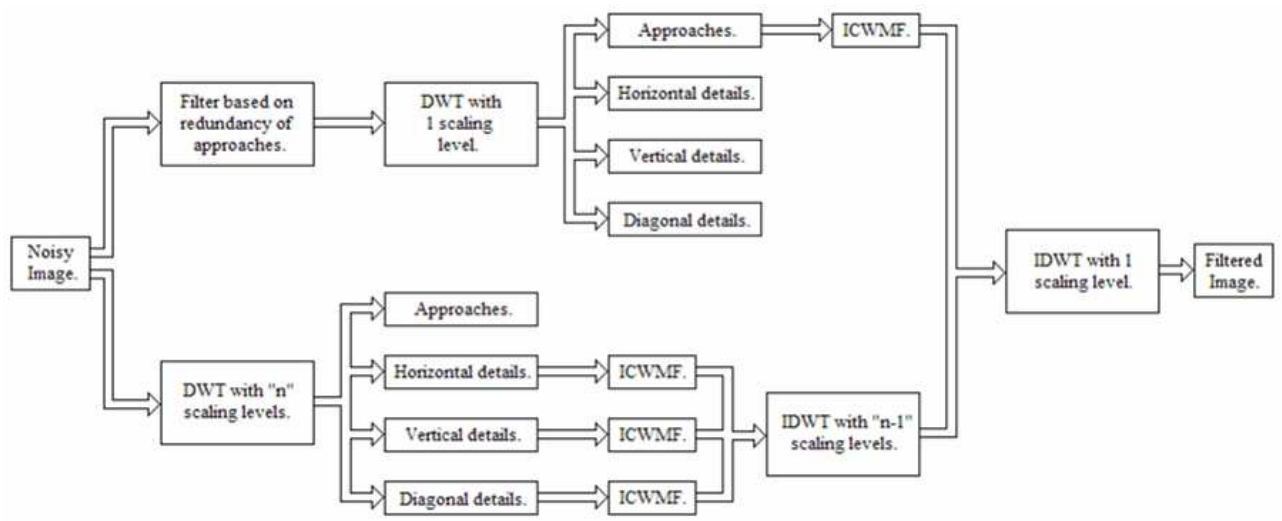

Fig. 1. Block diagram of the proposed filtering scheme of the Wavelet Domain Order Statistics Filter.

The first stage of the ICWMF that detects if a sample contains noise or not is given by:

$$
\sigma_{g s}^{2}(k)= \begin{cases}\tilde{\sigma}_{g s}^{2}(k), & \text { if } \lambda_{s} \geq \lambda_{t h} \\ \operatorname{med}_{c w}^{i}\left(\tilde{\sigma}_{g s}^{2}(j)\right), & \text { otherwise }\end{cases}
$$

where $\tilde{\sigma}_{g s}^{2}$ is the variance field estimated previously, $k$ is central sample in the filter window, $j$ is one of the $N$ sample contained into the window, $\lambda_{s}$ is the standard deviation of the preliminary estimate of the signal coefficients variances $\tilde{\sigma}_{s}^{2}(k)$ in each scale, $\lambda_{t h}=\sum_{s} \lambda_{s} 2^{-s} / \sum_{s} 2^{-s}$ is the discriminating threshold, $s$ is the scale used in the wavelet analysis, and $2^{-s}$ is the weighting function (Mahbubur Rahman \& Kamrul Hasan, 2003).

The Signal dependent rank order mean (SDROM) (Abreu et al., 1996), Adaptive Center Weighed Median (ACWM) (Chen \& Wu, 2001), and Median M-type KNN (Gallegos-Funes et al., 2007) filters were applied to the proposed filter as a first detection block. But the FIR Median Hybrid (FIRMH) filter (Astola \& Kuosmanen, 1997) was applied as a second detection block because this algorithm only constitutes the part of estimation of the noisy sample value (only if the sample was detected of this way) and the proposed filter can continue operating in all its sections in the same way. For this reason it is necessary to present the expression for the second detection block contained in the proposed filter structure (Mahbubur Rahman \& Kamrul Hasan, 2003):

$$
\begin{cases}\operatorname{med}\left(\widetilde{\sigma}_{g s}^{2}(j)\right) & \text { if } \tilde{\sigma}_{g s}^{2}(k) \leq \gamma \sigma_{n}^{2} \\ \widetilde{\sigma}_{g s}^{2}(k)+\operatorname{med}\left(\widetilde{\sigma}_{g s}^{2}(j)-\widetilde{\sigma}_{g s}^{2}(k)\right) & \text { otherwise }\end{cases}
$$

The proposed filter uses the median algorithm represented as $\operatorname{med}\left(\tilde{\sigma}_{g s}^{2}(j)\right)$ to estimate the value of the central sample in a filter window if the sample is detected as noisy. It is possible 
to use other estimation algorithm such as the FIR Median Hybrid Filter that retains more information about the image.

\subsection{Median M-type L-filter}

We propose to improve the robustness of $L$-filter by means of use of RM-estimator (11). The representation of L-filter is $\theta_{\mathrm{L}}=\sum_{i=1}^{N} a_{i} \cdot X_{(i)}$ where $X_{(i)}$ is the ordered data sample, $i=1, \ldots, N$, $a_{i}=\int_{i-1 / N}^{i / N} h(\lambda) d \lambda / \int_{0}^{1} h(\lambda) d \lambda$ are the weight coefficients, and $h(\lambda)$ is a probability density function (Kotropoulos \& Pitas, 2001).

To introduce the MM-estimator (11) in the scheme of $L$-filter, we present the ordered data sample of $L$-filter as function of an influence function (Gallegos-Funes et al., 2008),

$$
\hat{\theta}_{\mathrm{L}}=\sum_{i=1}^{N} a_{i} \cdot \psi\left(X_{i}\right) \cdot X_{i}
$$

where $N=(2 L+1)^{2}$ is the filtering window size, $\psi\left(X_{i}\right) \cdot X_{i}$ is the ordered data sample, $\psi(u)=\left\{\begin{array}{ll}c, & |u| \leq r \\ 0, & \text { otherwise }\end{array}\right.$ is the influence function, $c$ is a constant, and $r$ is connected with the range of $\psi(u)$.

Then, the non iterative MML filter can be obtained by the combination of $L$-filter (24) and the MM-estimator (11) (Gallegos-Funes et al., 2008),

$$
\hat{\theta}_{\mathrm{MML}}=\frac{\operatorname{MED}\left\{a_{i} \cdot\left[X_{i} \cdot \psi\left(X_{i}-\operatorname{MED}\{\vec{X}\}\right)\right]\right\}}{a_{\mathrm{MED}}}
$$

where $X_{i} \psi\left(X_{i}-\operatorname{MED}\{\vec{X}\}\right)$ are the selected pixels in accordance with the influence function used in a sliding filter window, the coefficients $a_{i}$ are computed using the Laplacian and Uniform distribution functions in $h(\lambda)$, and $a_{\mathrm{MED}}$ is the median of coefficients $a_{i}$ used as a scale constant.

To improve the properties of noise suppression of MML filter we use an impulsive noise detector (IND) (Aizenberg et al., 2003),

$$
\mathrm{IND}= \begin{cases}\text { Filtering, } & \text { if }[(D \leq s) \vee(D \geq N-s)] \wedge\left(\left|X_{c}-\operatorname{MED}(\vec{X})\right| \geq U\right) \\ X_{c}, & \text { otherwise }\end{cases}
$$

where $X_{c}$ is the central pixel in the filtering window, $s>0$ and $U \geq 0$ are thresholds, $N$ is the length of the data, and $D=\operatorname{rank}\left(X_{c}\right)$ is the rank of element $X_{c}$. The expressions $D \leq s$ and $D \geq N-s$ come from the fact that the difference between the ranks of the impulse and the median is usually large. In other words, the median is positioned in the center of data, and an impulse is usually positioned near one of its ends. The expression $\left|X_{c}-\operatorname{MED}(\vec{X})\right| \geq U$ has been specially developed for images that have very high corruption rate. Finally, if these conditions are true then we classify $X_{c}$ as corrupted. 


\subsection{Wavelet Domain Median M-type L-filter}

Figure 2 shows a block diagram of proposed Wavelet Domain Median M-type L (WDMML) filter (Varela-Benitez et al., 2007b). The proposed WDMML filter uses the Daubechie wavelets (Walker, 1999). We apply the proposed MML filter in the gray scale images of approaches and details obtained in the process of wavelet decomposition.
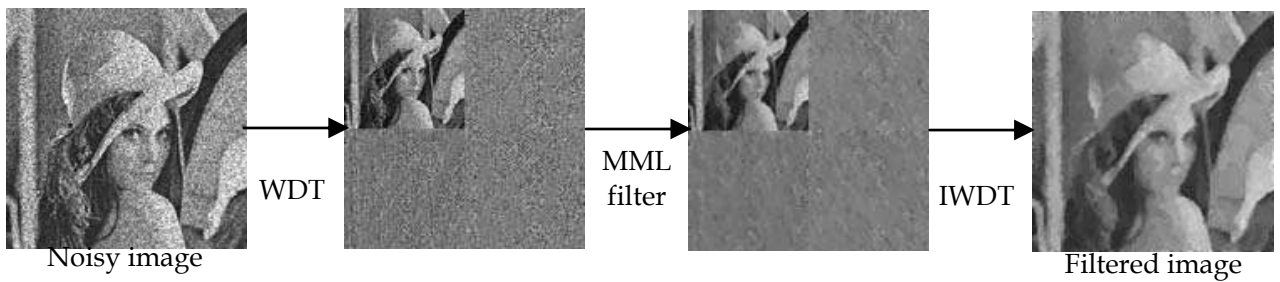

Fig. 2. Block diagram of proposed WDMML filter.

\section{Overall filtering performance}

The objective criteria used to compare the performance of noise suppression of various filters was the peak signal to noise ratio (PSNR) and for the evaluation of fine detail preservation the mean absolute error (MAE) was used (Astola \& Kuosmanen, 1997; Bovik 2000),

$$
\begin{gathered}
\text { PSNR }=10 \cdot \log \left[\frac{(255)^{2}}{\mathrm{MSE}}\right] \mathrm{dB} \\
\mathrm{MAE}=\frac{1}{M N} \sum_{i=0}^{M-1} \sum_{j=0}^{N-1}|f(i, j)-\hat{\theta}(i, j)|
\end{gathered}
$$

where $\mathrm{MSE}=\frac{1}{M N} \sum_{i=0}^{M-1} \sum_{j=0}^{N-1}[f(i, j)-\theta(i, j)]^{2}$ is the mean square error, $f(i, j)$ is the original image; $\hat{\theta}(i, j)$ is the restored image; and $M, N$ is the size of the image.

In the case of color image processing, we compute the mean chromaticity error (MCRE) for evaluation of chromaticity retention, and the normalized color difference (NCD) for quantification of color perceptual error (Plataniotis \& Venetsanopoulos, 2000):

$$
\begin{gathered}
\mathrm{MCRE}=\sum_{i=1}^{M_{1}} \sum_{j=1}^{M_{2}}\left\|p_{i, j}-\hat{p}_{i, j}\right\|_{L_{2}}^{2} / M_{1} M_{2} \\
\mathrm{NCD}=\sum_{i=1}^{M_{1}} \sum_{j=1}^{M_{2}}\left\|\Delta E_{L u v}(i, j)\right\|_{L_{2}} / \sum_{i=1}^{M_{1}} \sum_{j=1}^{M_{2}}\left\|E_{L u v}^{*}(i, j)\right\|_{L_{2}}
\end{gathered}
$$

where $p_{i, j}$ and $\hat{p}_{i, j}$ are the intersection points of $f(i, j)$ and $\hat{\theta}(i, j)$ with the plane defined by the Maxwell triangle, respectively, $\left\|\Delta E_{L u v}(i, j)\right\|_{L_{2}}=\left[\left(\Delta L^{*}(i, j)\right)^{2}+\left(\Delta u^{*}\right)^{2}+\left(\Delta v^{*}\right)^{2}\right]^{1 / 2}$ is the 
norm of color error, $\Delta L^{*}, \Delta u^{*}$, and $\Delta v^{*}$ are the difference in the $L^{*}, u^{*}$, and $v^{*}$ components, respectively, between the two color vectors that present the filtered image and uncorrupted original one for each a pixel $(i, j)$ of an image, $\left\|E_{L u v}^{*}(i, j)\right\|_{L_{2}}=\left[\left(L^{*}\right)^{2}+\left(u^{*}\right)^{2}+\left(v^{*}\right)^{2}\right]^{1 / 2}$ is the norm or magnitude of the uncorrupted original image pixel vector in the $L^{*} u^{*} v^{*}$ space, and $\|\cdot\|_{L_{2}}$ is the $\mathrm{L}_{2}$-vector norm.

\subsection{Noise suppression in gray scale images and video sequences}

The described 3×3 MMKNN, 3×3 WMKNN, 3×3 ABSTMKNN, 3×3 MOODMKNN, and 3×3 MML filters with different influence functions have been evaluated, and their performance has been compared with $3 \times 3$ weighted median (WM) (Bovik, 2000), 3×3 tri-state median (TSM) (Chen et al., 1999), 3×3 adaptive center weighted median (ACWM) (Chen \& Wu, 2001), 3×3 rank order mean (ROM) (Abreu et al., 1996), 3×3 minimum-maximum exclusive mean (MMEM) (WeiLu \& Ja-Chen, 1997), 3x3 Local Linear Minimum Mean Square Error (LMMSE) (Özkan et al., 1993), 3x3 K-Nearest Neighbor (KNN) (Astola \& Kuosmanen, 1997), 3x3 Ansari-Bradley-SiegelTukey (ABST) (Hampel et al., 1986), 3×3 Normalized Least Mean Squares L (NLMS-L) (Kotropoulos \& Pitas, 1996), 3x3 Sampled-Function Weighted Order (SFWO) (Öten \& De Figueiredo, 2002), and Modified Frost (MFrost) (Lukin et al., 1998) filters. The reason for choosing these filters to compare them with the proposed ones is that their performances have been compared with various known filters and their advantages have been demonstrated. The runtime analysis of various filters was conducted for different images using Texas Instruments DSP TMS320C6701 (Kehtarnavaz \& Keramat, 2001; Texas Instruments, 1998).

To determine the impulsive noise suppression properties of various filters the $256 \times 256$ standard test grayscale images "Airfield", "Goldhill", and "Lena" were corrupted with an occurrence rate of $20 \%$ of impulsive noise and the results obtained by the mentioned filters are presented in Table 2. One can see from the Table 2 that the proposed MMKNN and WMKNN filters have better performances in terms of PSNR and MAE criteria in comparison with the filters used as comparative in the most of cases. The processing time is given in seconds and includes the duration of data acquisition, processing and storing of data.

The results reveal that the processing time values of the MMKNN filter are larger than WM, and MMEM filters but less in comparison with ACWM, LMMSE, and ROM filters and have about the same values compared to the TSM filter. The MMKNN filter with Andrew's and Bernoulli influence functions take more time than when other influence functions are used depending on the filter parameters values. For the ROM filter the processing time does not include the time for deriving weighting coefficients during the training stage and then used in this filtering scheme. The time used in its training procedure is $0.035 \mathrm{~s}$ approximately. The processing time performance of the MMKNN filter depends on the image to process and almost does not vary for different noise levels; these values also depend on the complex calculation of the influence functions and parameters of the proposed filter. The proposed MMKNN algorithm can process from 16 to 19 images of 256x256 pixels per second. In the case of WMKNN filter we observe that its processing time is larger than MMKNN filter. The WMKNN algorithm can process from 10 to 14 images of 256x256 pixels per second.

In Figure 3 we present the processed images for the test image "Lena" explaining the impulsive noise suppression according to the Table 2. A zoomed-in section (upright) of each image is displayed in order to view the details. 


\begin{tabular}{|c|c|c|c|c|c|c|c|c|c|}
\hline \multirow{2}{*}{ Algorithm } & \multicolumn{3}{|c|}{ Airfield } & \multicolumn{3}{c|}{ Goldhill } & \multicolumn{3}{c|}{ Lena } \\
\cline { 2 - 11 } & PSNR & MAE & TIME & PSNR & MAE & & PSNR & MAE & \\
\hline WM & 22.40 & 10.67 & 0.0203 & 24.21 & 9.96 & 0.0203 & 23.58 & 8.67 & 0.0203 \\
\hline TSM & 21.13 & 13.44 & 0.0547 & 23.11 & 12.43 & 0.0547 & 23.56 & 10.80 & 0.0547 \\
\hline ACWM & 22.97 & 10.57 & 0.2299 & 24.84 & 10.43 & 0.2299 & 25.56 & 8.75 & 0.2299 \\
\hline ROM & 23.08 & 10.42 & 0.0750 & 24.82 & 10.57 & 0.0750 & 25.20 & 9.11 & 0.0750 \\
\hline MMEM & 22.69 & 12.23 & 0.0406 & 24.16 & 11.09 & 0.0406 & 24.52 & 9.46 & 0.0406 \\
\hline LMMSE & 23.03 & 11.24 & 0.0750 & 24.15 & 11.08 & 0.0750 & 24.59 & 9.95 & 0.0751 \\
\hline MMKNN (S) & 23.21 & 10.45 & 0.0515 & 25.45 & 9.58 & 0.0517 & 26.38 & 7.12 & 0.0515 \\
\hline MMKNN (H) & 23.24 & 10.42 & 0.0521 & 25.50 & 9.50 & 0.0524 & 26.33 & 7.07 & 0.0521 \\
\hline MMKNN (A) & 23.23 & 10.44 & 0.0566 & 25.48 & 9.53 & 0.0573 & 26.36 & 7.12 & 0.0557 \\
\hline MMKNN (T) & 23.23 & 10.45 & 0.0528 & 25.50 & 9.56 & 0.0555 & 26.32 & 7.13 & 0.0528 \\
\hline MMKNN (B) & 23.24 & 10.46 & 0.0593 & 25.50 & 9.56 & 0.0599 & 26.31 & 7.14 & 0.0588 \\
\hline WMKNN (S) & 22.82 & 10.82 & 0.0686 & 25.29 & 9.97 & 0.0751 & 25.66 & 7.60 & 0.0757 \\
\hline WMKNN (H) & 22.72 & 10.86 & 0.0736 & 25.19 & 10.00 & 0.0826 & 25.45 & 7.67 & 0.0814 \\
\hline WMKNN (A) & 22.79 & 10.84 & 0.0920 & 25.36 & 9.92 & 0.0979 & 25.68 & 7.56 & 0.0944 \\
\hline WMKNN (T) & 22.30 & 11.23 & 0.0753 & 24.95 & 10.46 & 0.0804 & 24.88 & 8.11 & 0.0775 \\
\hline WMKNN (B) & 22.26 & 11.29 & 0.0695 & 24.41 & 10.40 & 0.0861 & 24.79 & 8.05 & 0.0838 \\
\hline
\end{tabular}

Table 2. PSNR in $\mathrm{dB}, \mathrm{MAE}$, and Processing time values for different images corrupted by $20 \%$ of impulsive noise obtained by different filters.

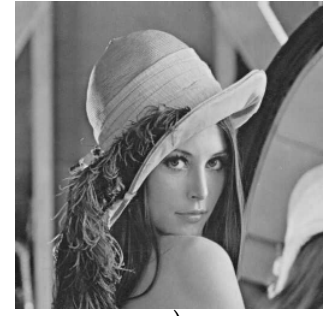

a)

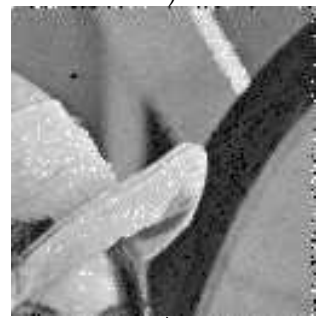

e)

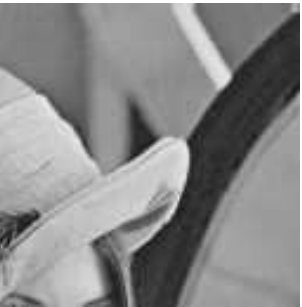

b)

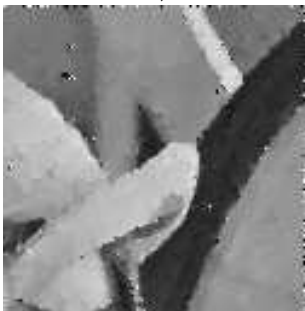

f)

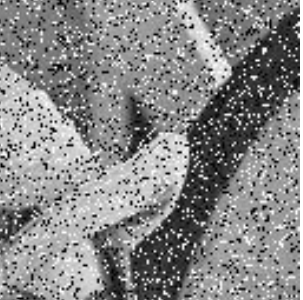

c)

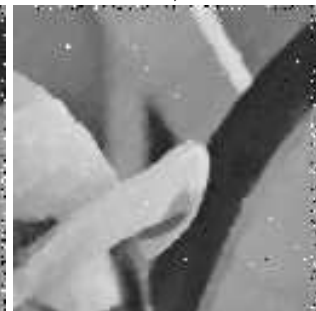

g)

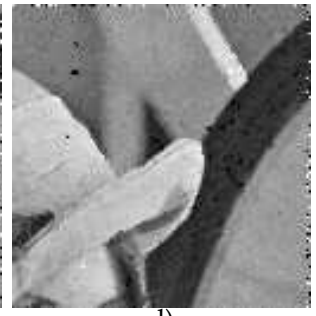

d)

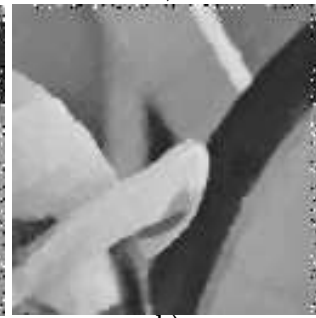

h)

Fig. 3. Subjective visual qualities of a part of image "Lena", a) Original image, b) Zoomed-in section (upright) of (a), c) Degraded image with $20 \%$ of impulsive noise of (b), d) Restored image with the ACWM filter of (b), e) Restored image with the ROM filter of (b), f) Restored image with the LMMSE filter of (b), g) Restored image with the MMKNN (H) filter of (b), h) Restored image with the MM-KNN (B) filter of (b). 
Table 3 shows the performance results in the grayscale image "Peppers" degraded with 20\% of impulsive noise. We observe that the processing time values of ABSTMKNN and MOODMKNN filters are larger than the most comparison filters but the proposed filters consistently outperform other filters by balancing the tradeoff between noise suppression and detail preservation. Figure 4 depicts that the restored image by ABSTMKNN method appears to have a very good subjective quality in comparison with other methods.

\begin{tabular}{|c|c|c|c|}
\hline Algorithm & PSNR dB & MAE & TIME \\
\hline KNN & 18.83 & 24.32 & 0.021380 \\
\hline ABST & 22.61 & 11.10 & 0.038395 \\
\hline WM & 24.68 & 7.81 & 0.020341 \\
\hline ACWM & 25.18 & 9.21 & 0.229951 \\
\hline ROM & 25.04 & 9.62 & 0.075008 \\
\hline MMEM & 24.40 & 9.67 & 0.040618 \\
\hline LMMSE & 24.75 & 9.70 & 0.075140 \\
\hline ABSTMKNN (S) & 25.85 & 7.55 & 0.063876 \\
\hline ABSTMKNN (H) & 25.62 & 7.75 & 0.063787 \\
\hline ABSTMKNN (A) & 25.95 & 7.57 & 0.074301 \\
\hline ABSTMKNN (T) & 25.51 & 7.75 & 0.063151 \\
\hline ABSTMKNN (B) & 25.46 & 7.75 & 0.067383 \\
\hline MOODMKNN (S) & 25.98 & 7.55 & 0.066725 \\
\hline MOODMKNN (H) & 25.62 & 7.75 & 0.067295 \\
\hline MOODMKNN (A) & 25.98 & 7.57 & 0.076487 \\
\hline MOODMKNN (T) & 25.67 & 7.64 & 0.068421 \\
\hline MOODMKNN (B) & 25.58 & 7.66 & 0.067413 \\
\hline
\end{tabular}

Table 3. Performance results for image "Peppers".

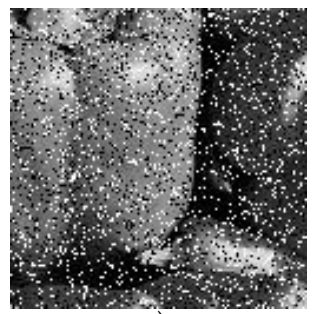

a)

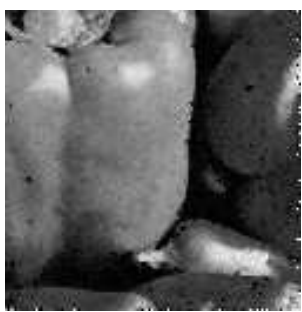

b)

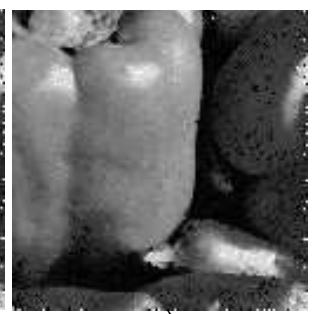

c)

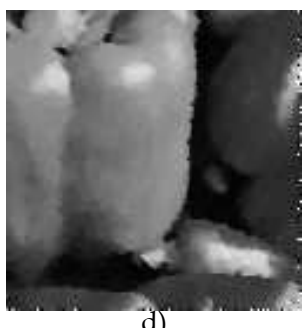

d)

Fig. 4. Results for a part of "Peppers" image, a) Degraded image with 20\% of impulse noise, b) Restored image by ACWM filter, c) Restored image by the ROM filter, d) Restored image by the ABSTM-KNN (S) filter.

The MML filter was implemented with Laplacian (L) and Uniform (U) distribution functions, and with (D) and without (ND) impulsive noise detector. Table 1 shows the performance results for "Lena" image degraded with $5 \%$ of impulsive noise and $\sigma^{2}=0.05$ of speckle noise. From Table 4, the proposed filter provides better noise suppression and detail preservation than other filters in the most of cases. The processing time of MML filter is less than filters used as comparative, and it takes less time when the impulsive noise detector is 
used. Figure 5 exhibits the filtered images in the case of $20 \%$ of impulsive noise. The restored image by proposed MML filter appears to have good subjective quality.

To demonstrate the performances of the proposed MML filtering scheme we apply it for filtering of the SAR images, which naturally have speckle noise. The results of such a filtering are presented in the Figure 6 in the case of the image "Pentagon". It is possible to see analyzing the filtering images that speckle noise can be efficiently suppressed, while the sharpness and fine feature are preserved using the proposed filter in comparison with other filters proposed in the references.

\begin{tabular}{|l|c|c|c|c|c|c|}
\hline \multirow{2}{*}{ Filters } & \multicolumn{3}{|c|}{ Impulsive noise $=5 \%$} & \multicolumn{3}{c|}{ Speckle noise $=0.05$} \\
\cline { 2 - 7 } & PSNR & MAE & TIME & PSNR & MAE & TIME \\
\hline ACWM & 27.73 & 7.35 & 0.2299 & 19.96 & 20.34 & 0.2299 \\
\hline ROM & 27.49 & 7.64 & 0.1050 & 22.82 & 20.96 & 0.1050 \\
\hline MFrost & 23.87 & 12.69 & 0.1004 & 24.56 & 10.99 & 0.1004 \\
\hline NLMS-L & 24.24 & 11.57 & 0.1835 & 21.59 & 21.54 & 0.1835 \\
\hline SFWO (L) & 24.94 & 8.38 & 0.1310 & 22.10 & 14.37 & 0.1310 \\
\hline SFWO (U) & 15.76 & 32.04 & 0.1310 & 22.53 & 12.84 & 0.1310 \\
\hline MML (A,L,ND) & 27.01 & 7.61 & 0.0815 & 22.78 & 14.21 & 0.0815 \\
\hline MML (A,U,ND) & 28.03 & 6.13 & 0.0815 & 24.61 & 10.92 & 0.0815 \\
\hline MML (T,L,ND) & 26.93 & 7.62 & 0.0796 & 22.63 & 14.44 & 0.0796 \\
\hline MML (T,U,ND) & 28.29 & 5.76 & 0.0796 & 24.79 & 10.63 & 0.0796 \\
\hline MML (H,L,ND) & 27.37 & 6.92 & 0.0804 & 23.12 & 13.53 & 0.0804 \\
\hline MML (H,U,ND) & 28.40 & 5.56 & 0.0804 & 24.86 & 10.53 & 0.0804 \\
\hline MML (A,L,D) & 28.62 & 6.01 & 0.0684 & 23.38 & 13.10 & 0.0685 \\
\hline MML (A,U,D) & 29.10 & 5.51 & 0.0684 & 24.60 & 11.00 & 0.0684 \\
\hline MML (T,L,D) & 28.59 & 6.04 & 0.0652 & 23.40 & 13.03 & 0.0652 \\
\hline MML (T,U,D) & 29.23 & 5.34 & 0.0651 & 24.63 & 10.96 & 0.0652 \\
\hline MML (H,L,D) & 28.75 & 5.83 & 0.0790 & 23.61 & 12.67 & 0.0790 \\
\hline MML (H,U,D) & 29.33 & 5.16 & 0.0790 & 24.86 & 10.53 & 0.0790 \\
\hline
\end{tabular}

Table 4. Performance results in image "Lena" obtained by different filters,

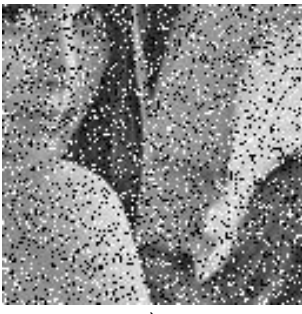

a)

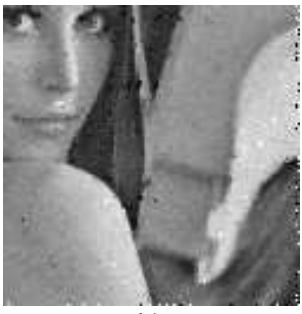

b)

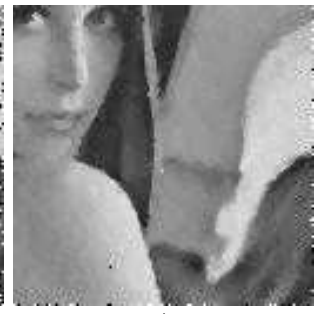

c)

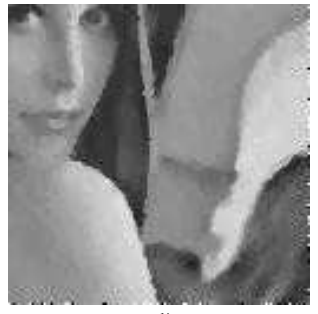

d)

Fig. 5. Filtered images with $20 \%$ of impulsive noise: a) Degraded image, b) ACWM, c) ROM, d) $\operatorname{MML}(\mathrm{A}, \mathrm{L}, \mathrm{ND})$.

Table 5 shows the performance results in terms of PSNR in dB and MAE for the image "Lena" degraded with 0.1 of variance of speckle noise and free of noise by use the WDMML 
$(\mathrm{S}, \mathrm{ND}, \mathrm{L})$ filter in approaches (A) and details (D) with the wavelets db1, db2, db3, and db4 with one (1) and two (2) levels of decomposition. From this Table one can see that the proposed WDMML filter provides better speckle noise suppression and detail preservation in comparison with the MML filter in the spatial domain in the most of cases. Figure 7 presents the visual results to apply the proposed filter with one and two decomposition levels in the image "Peppers". One can see from Figure 7 that the proposed WDMML filter outperforms the MML filter in the case of speckle noise.

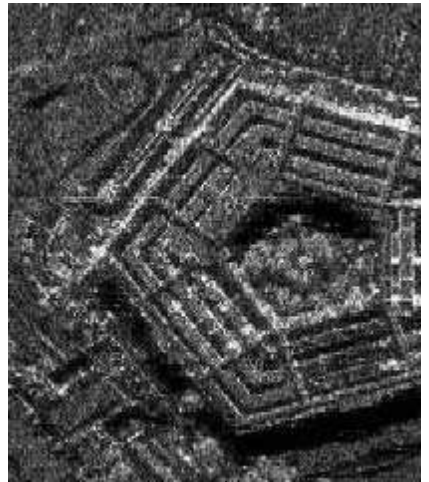

a)

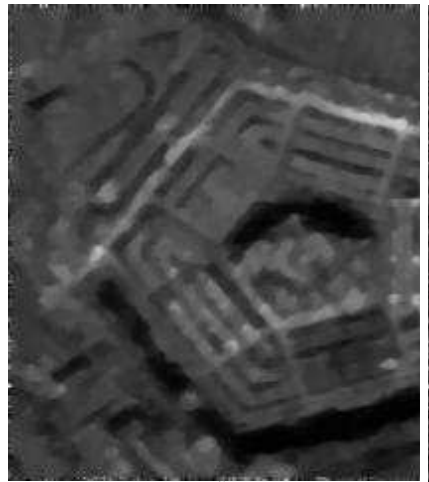

d)

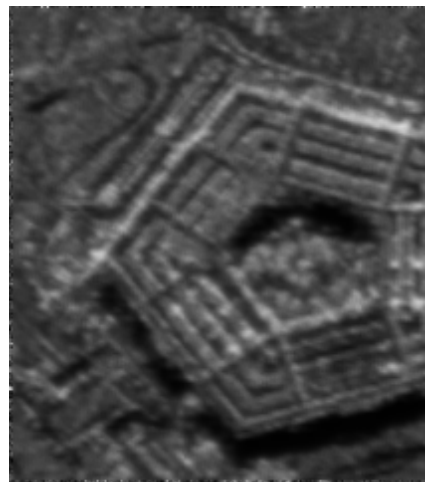

b)

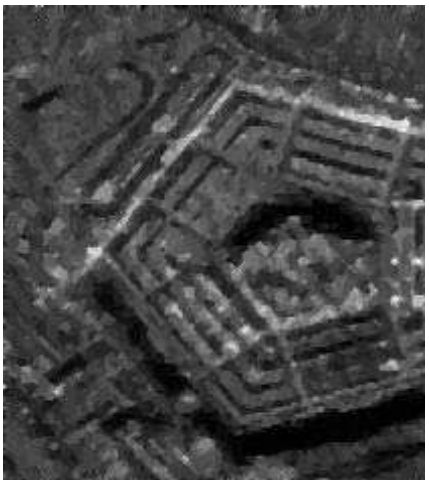

e)

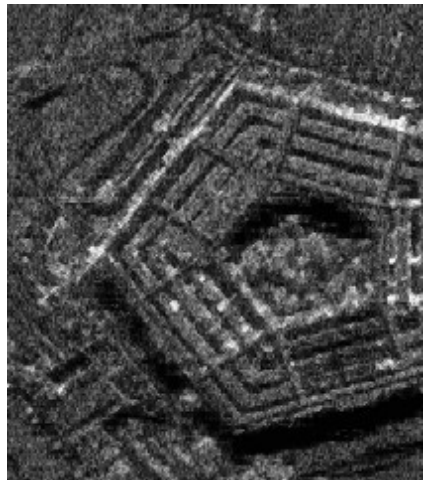

C)

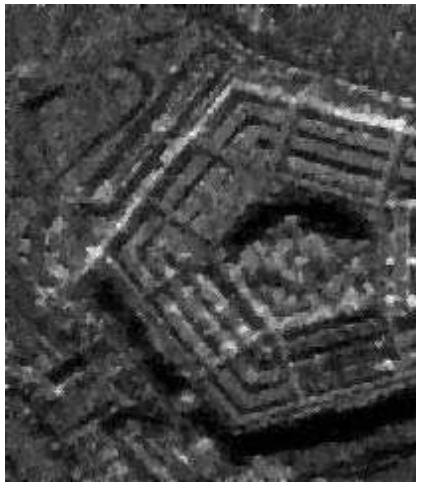

f)

Fig. 6. Comparative results of despeckled SAR image. a) Original image "Pentagon", resolution 1m, source Sandia National Lab., b) Despeckled image with MFrost filter, c) Despeckled image with the ROM filter, d) Despeckled image with the SFWO filter, e) Despeckled image with the MML filter (S, ND, L), f) Despeckled image with the MML filter $(\mathrm{S}, \mathrm{D}, \mathrm{L})$

We also propose to apply the proposed filters to video signals. We process a real video sequence to demonstrate that the proposed method potentially could provide a real-time solution to quality video transmission. We investigate a QCIF (Quarter Common Intermediate Format) video sequence. This picture format uses 176x144 luminance pixels per frame and velocity from 15 to 30 frames per second. In the case of this test we used one frame of the video sequence "carphone", that was corrupted by $20 \%$ of impulsive noise. The 
PSNR, MAE and processing time performances are depicted in Table 6. The restored frames are displayed in Figure 8 by using the ACWM, LMMSE, and MMKNN $(\mathrm{H})$ filters. From the simulation results we observe that the proposed MMKNN and ABSTMKNN filters can process up to 33 frames of QCIF video format suppressing the impulsive noise and providing the detail preservation in real-time applications.

\begin{tabular}{|l|c|c|c|c|}
\hline \multirow{2}{*}{ Filters } & \multicolumn{2}{|c|}{ Free noise } & \multicolumn{2}{c|}{$\sigma^{2}=0.1$} \\
\cline { 2 - 5 } & PSNR & MAE & PSNR & MAE \\
\hline MML (S,ND,L) & 29.62 & 3.78 & 22.95 & 13.24 \\
\hline WDMML (S,ND,db1,A,1) & 27.84 & 5.09 & 23.35 & 12.24 \\
\hline WDMML (S,ND,db1,D,1) & 31.46 & 3.24 & 20.53 & 18.78 \\
\hline WDMML (S,ND,db2,A,1) & 27.90 & 5.11 & 23.60 & 12.15 \\
\hline WDMML (S,ND,db2,D,1) & 32.26 & 3.05 & 20.69 & 18.00 \\
\hline WDMML (S,ND,db3,A,1) & 27.92 & 5.24 & 24.02 & 11.77 \\
\hline WDMML (S,ND,db3,D,1) & 32.70 & 2.97 & 20.79 & 17.99 \\
\hline WDMML (S,ND,db4,A,1) & 27.87 & 5.27 & 24.33 & 11.28 \\
\hline WDMML (S,ND,db4,D,1) & 33.00 & 2.92 & 20.90 & 18.11 \\
\hline WDMML (S,ND,db1,A,2) & 24.83 & 8.32 & 22.46 & 13.57 \\
\hline WDMML (S,ND,db1,D,2) & 27.48 & 5.73 & 22.66 & 13.93 \\
\hline WDMML (S,ND,db2,A,2) & 25.40 & 7.61 & 22.94 & 12.85 \\
\hline WDMML (S,ND,db2,D,2) & 28.37 & 5.34 & 23.21 & 13.15 \\
\hline WDMML (S,ND,db3,A,2) & 25.24 & 7.89 & 23.14 & 12.59 \\
\hline WDMML (S,ND,db3,D,2) & 28.39 & 5.42 & 23.49 & 12.90 \\
\hline WDMML (S,ND,db4,A,2) & 25.06 & 8.21 & 23.38 & 12.47 \\
\hline WDMML (S,ND,db4,D,2) & 28.29 & 5.46 & 23.69 & 12.73 \\
\hline
\end{tabular}

Table 5. Performance results in the image "Lena" obtained by the use of WDMML filter.

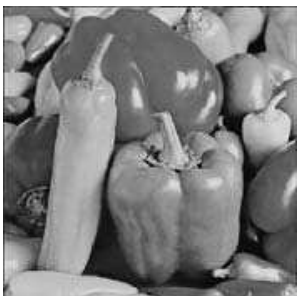

a)

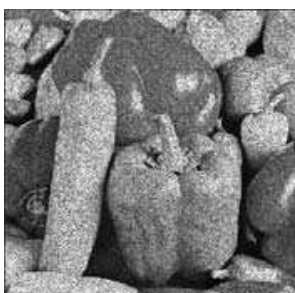

b)

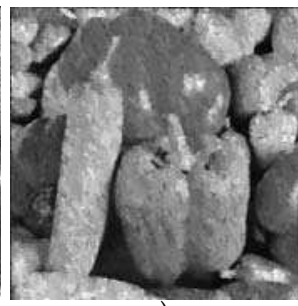

C)

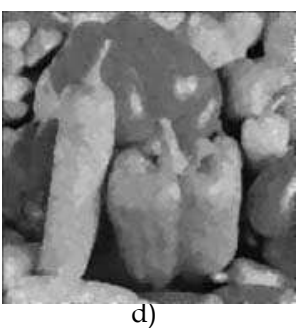

d)

Fig. 7. Visual results in the image Peppers, a) Original image, b) Degraded image with 0.1 of variance of speckle noise, $c)$ Restored image with MML (S,L,ND) filter, d) Restored image with WDMML filter (S,L,ND,db2,A,1).

\subsection{Noise suppression in color images and video sequences}

The proposed MMKNN, WMKNN, and ABSTMKNN filters were adapted to work in color image and video processing. Now, the proposed Vector RMKNN filters are called as VMMKNN, VWMKNN, and VABSTMKNN filters. These filters have been evaluated, and their performances have been compared with vector median (VMF) (Plataniotis \& 


\begin{tabular}{|c|c|c|c|}
\hline \multirow{2}{*}{ Algorithm } & \multicolumn{3}{|c|}{ “Carphone" Frame } \\
\cline { 2 - 4 } & PSNR & MAE & Time \\
\hline WM & 23.83 & 9.80 & 0.009977 \\
\hline TSM & 21.53 & 13.24 & 0.023730 \\
\hline ACWM & 24.34 & 9.79 & 0.093865 \\
\hline MMEM & 23.60 & 11.11 & 0.018021 \\
\hline LMMSE & 24.15 & 10.04 & 0.034716 \\
\hline MMKNN (S) & 24.77 & 9.08 & 0.023773 \\
\hline MMKNN (H) & 24.76 & 9.10 & 0.024018 \\
\hline MMKNN (A) & 24.75 & 9.06 & 0.025717 \\
\hline MMKNN (T) & 24.74 & 9.16 & 0.024166 \\
\hline MMKNN (B) & 24.78 & 9.15 & 0.026760 \\
\hline ABSTMKNN (S) & 25.07 & 9.44 & 0.027068 \\
\hline ABSTMKNN (H) & 24.79 & 9.66 & 0.027932 \\
\hline ABSTMKNN (A) & 25.08 & 9.48 & 0.029762 \\
\hline ABSTMKNN (T) & 24.78 & 9.62 & 0.026815 \\
\hline ABSTMKNN (B) & 24.88 & 9.62 & 0.028440 \\
\hline
\end{tabular}

Table 6. PSNR values in $\mathrm{dB}, \mathrm{MAE}$, and processing time for different filters in a frame of video sequence "Carphone".

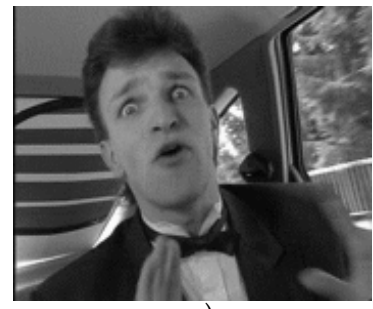

a)

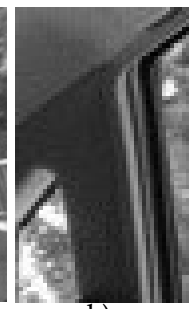

b)

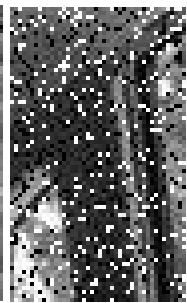

C)

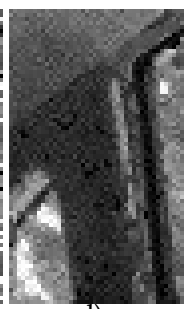

d)

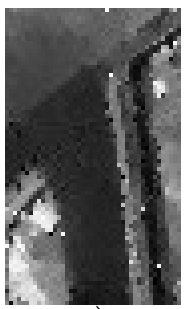

e)

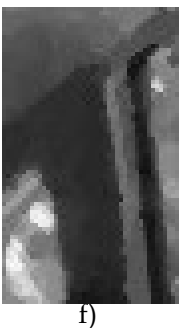

f)

Fig. 8. Subjective visual qualities of a restored frame "Carphone" produced by different filters, a) Original frame, b) Zoomed-in section (upright) of (a), c) Degraded frame with 20\% of impulsive noise of (b), d) Restored frame with the ACWM filter of (b), e) Restored frame with the LMMSE filter of (b), f) Restored frame with the MMKNN (H) filter of (b).

Venetsanopoulos, 2000), $\alpha$-trimmed mean ( $\alpha-\mathrm{TMF})$, basic vector directional (BVDF), generalized vector directional (GVDF), adaptive GVDF (AGVDF), double window GVDF (GVDF_DW), and multiple non-parametric (MAMNFE) (Trahanias et al., 1996; Plataniotis et al., 1997) filters.

The implementation of filters were realized on the DSP TMS320C6711 (Kehtarnavaz \& Keramat, 2001; Texas Instruments, 1998) to demonstrate that the proposed filters potentially could provide a real-time solution to quality video transmission.

The 320 X320 "Lena" color image was corrupted by $20 \%$ of impulsive noise. Table 7 shows that the performance criteria are often better for the proposed filters in comparison when other filters are used. Figure 9 exhibits the processed images for test image "Lena" explaining the impulsive noise suppression, and presenting the original image "Lena", image corrupted with noise probability occurrence of $20 \%$ for each color channel, and 
exhibiting the filtering results produced by the MAMNFE and VMMKNN filters, respectively. The proposed VMMKNN filtering appears to have a better subjective quality in comparison with MAMNFE filtering.

\begin{tabular}{|c|c|c|c|c|c|}
\hline Algorithm & PSNR & MAE & MCRE & NCD & TIME \\
\hline VMF & 21.15 & 10.73 & 0.035 & 0.038 & 0.039 \\
\hline$\alpha$-TMF & 20.86 & 14.97 & 0.046 & 0.049 & 0.087 \\
\hline BVDF & 20.41 & 12.72 & 0.043 & 0.045 & 0.065 \\
\hline GVDF & 20.67 & 11.18 & 0.038 & 0.040 & 0.264 \\
\hline AGVDF & 22.01 & 11.18 & 0.028 & 0.036 & 0.620 \\
\hline GVDF_DW & 22.59 & 10.09 & 0.028 & 0.039 & 0.721 \\
\hline MAMNFE & 22.67 & 9.64 & 0.027 & 0.035 & 0.832 \\
\hline VMMKNN (S) & 23.15 & 10.00 & 0.033 & 0.034 & 0.296 \\
\hline VMMKNN (A) & 23.07 & 10.01 & 0.033 & 0.035 & 0.199 \\
\hline VMMKNN (H) & 23.05 & 10.04 & 0.033 & 0.035 & 0.199 \\
\hline VWMKNN (S) & 22.99 & 10.13 & 0.033 & 0.035 & 0.435 \\
\hline VWMKNN (A) & 23.00 & 10.08 & 0.033 & 0.035 & 0.756 \\
\hline VWMKNN (H) & 22.99 & 10.09 & 0.033 & 0.035 & 0.398 \\
\hline VABSTMKNN (S) & 22.99 & 10.13 & 0.033 & 0.035 & 0.286 \\
\hline VABSTMKNN (A) & 22.99 & 10.13 & 0.033 & 0.035 & 0.320 \\
\hline VABSTMKNN (H) & 23.01 & 10.07 & 0.033 & 0.035 & 0.264 \\
\hline
\end{tabular}

Table 7. Comparative restoration results for $20 \%$ of impulsive noise for color image "Lena".

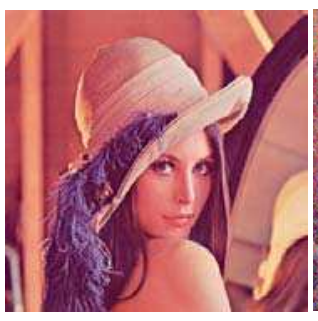

a)

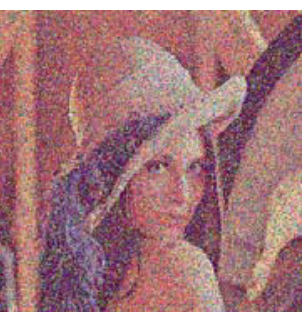

b)

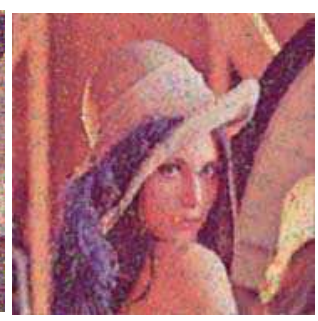

c)

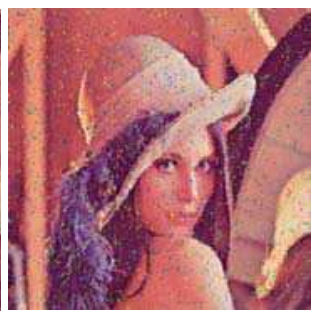

d)

Fig. 9. Subjective visual qualities of restored color image "Lena", a) Original test image "Lena", b) Input noisy image with 20\% of impulsive noise, c) MAMNFE filtering image, and d) Proposed VMMKNN (S) filtered image.

We use one frame of the video color sequence "Miss America", which was corrupted by $15 \%$ of impulsive noise. One can see in Table 8 that the performance criteria are often better for the proposed VMMKNN, VWMKNN, and VABSTMKNN filters in comparison when other filters are used in the most of cases. Figure 10 exhibits the processed frames for test image "Miss America" explaining the impulsive noise suppression. The restored frame with VMMKNN filter appears to have a better subjective quality in comparison with MAMNFE filter that has the better performance among the known color filters.

The processing time performance of the VRMKNN filters depends on the image to process and almost does not vary for different noise levels. These values also depend on the complex calculation of the influence functions and parameters of the proposed filters. From 
Tables 7 and 8, one can see that the proposed algorithms can process, in the case of color images of size 320x320 pixels, up to 5 images per second, and in case of QCIF video up to 11 color frames per second in comparison with MAMNFE filter (the best comparative filter) that can process one image per second or 3 frames per second.

\begin{tabular}{|c|c|c|c|c|c|}
\hline Algorithm & PSNR & MAE & MCRE & NCD & TIME \\
\hline VMF & 25.54 & 5.38 & 0.0371 & 0.0332 & 0.0153 \\
\hline$\alpha$-TMF & 24.47 & 6.54 & 0.0589 & 0.0251 & 0.0206 \\
\hline BVDF & 22.45 & 7.68 & 0.0379 & 0.0329 & 0.1768 \\
\hline GVDF & 23.56 & 9.12 & 0.0362 & 0.0308 & 0.1869 \\
\hline AGVDF & 26.97 & 5.24 & 0.0308 & 0.0302 & 0.2106 \\
\hline GVDF_DW & 26.88 & 5.95 & 0.0311 & 0.0249 & 0.7205 \\
\hline MAMNFE & 27.01 & 5.82 & 0.0390 & 0.0270 & 0.3219 \\
\hline VMMKNN (S) & 28.20 & 3.86 & 0.0312 & 0.0140 & 0.1109 \\
\hline VMMKNN (A) & 28.04 & 3.91 & 0.0317 & 0.0143 & 0.0898 \\
\hline VMMKNN (H) & 28.14 & 3.90 & 0.0315 & 0.0144 & 0.0917 \\
\hline VWMKNN (S) & 27.27 & 4.48 & 0.0336 & 0.0234 & 0.2662 \\
\hline VWMKNN (A) & 26.10 & 5.10 & 0.0372 & 0.0272 & 0.4599 \\
\hline VWMKNN (H) & 26.05 & 5.05 & 0.0369 & 0.0271 & 0.2912 \\
\hline VABSTMKNN (S) & 27.75 & 4.46 & 0.0336 & 0.0243 & 0.0929 \\
\hline VABSTMKNN (A) & 27.56 & 4.68 & 0.0349 & 0.0253 & 0.2066 \\
\hline VABSTMKNN (H) & 27.49 & 4.71 & 0.0350 & 0.0255 & 0.1194 \\
\hline
\end{tabular}

Table 8. Comparative restoration results for $15 \%$ impulsive noise for a color frame of "Miss America"

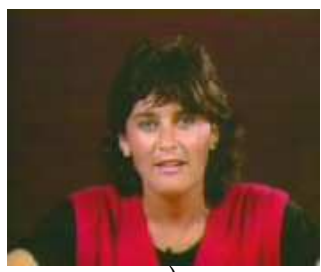

a)

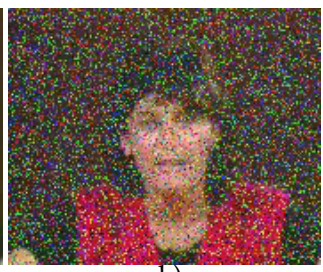

b)

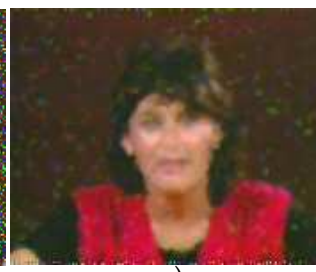

C)

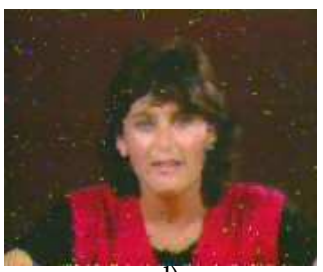

d)

Fig. 10. Subjective visual qualities of restored color frame "Miss America", a) Original test frame "Miss America", b) Input noisy frame with 15\% of impulsive noise, c) MAMNFE filtered frame, and d) Proposed VMMKNN filtered frame (A).

The proposed Wavelet Redundancy of Approaches (WRAF), Wavelet Iterative Center Weighted Median using Redundancy of Approaches (WICWMRAF), Wavelet Signal Dependent Rank-Ordered Mean (WSDROMF), Wavelet Adaptive Center Weighed Median (WACWMF), Wavelet Median M-type K-Nearest Neighbor (WMMKNNF), and Wavelet FIR Median Hybrid (WFIRMHF) Filters were compared with the Wavelet Iterative Median (WIMF) and Wavelet Iterative Center Weighted Median (WICWMF) (Mahbubur Rahman \& Kamrul Hasan, 2003) filters in terms of PSNR, MAE, MCRE and NCD to demonstrate the good quality of color imaging of the proposed filters in both an objective and subjective sense. 
Table 9 presents the performance results by means of use different filters in the $512 \times 512$ image "Lena" degraded with $20 \%$ of impulsive noise and with 0.2 of variance of speckle noise. From these results we observe that the proposed filters provide better impulsive and speckle noise suppression, detail preservation, and color retention in comparison with the traditional filters in the Wavelet domain. Figure 11 shows the subjective visual quantities of a restored zoom part of the color image "Lena" degraded with 0.2 of variance of speckle noise. Figure 12 presents the visual results in a part of "Mandrill" image produced by the WMMKNNF.

\begin{tabular}{|c|c|c|c|c|c|c|c|c|}
\hline Filters & \multicolumn{4}{|c|}{ 20\% of impulsive noise } & \multicolumn{3}{c|}{0.2 of variance of speckle noise } \\
\hline & PSNR & MAE & MCRE & NCD & PSNR & MAE & MCRE & NCD \\
\hline WIMF & 40.6734 & 24.7969 & 0.0172 & 0.3294 & 43.7164 & 22.2675 & 0.0138 & 0.2617 \\
\hline WICWMF & 40.6734 & 24.7969 & 0.0172 & 0.3294 & 43.7164 & 22.2675 & 0.0138 & 0.2617 \\
\hline WRAF & 44.4936 & 20.8426 & 0.0117 & 0.2718 & 48.0338 & 18.2333 & 0.0093 & 0.2062 \\
\hline WICWMRAF & 50.6952 & 15.8213 & 0.0063 & 0.1911 & 53.8602 & 14.1506 & 0.0056 & 0.1489 \\
\hline WSDROMF & 50.6952 & 15.8213 & 0.0063 & 0.1911 & 53.8602 & 14.1506 & 0.0056 & 0.1489 \\
\hline WACWMF & 50.6952 & 15.8213 & 0.0063 & 0.1911 & 53.8602 & 14.1507 & 0.0056 & 0.1489 \\
\hline WMMKNNF & 50.6953 & 15.8211 & 0.0063 & 0.1911 & 53.8603 & 14.1506 & 0.0056 & 0.1489 \\
\hline WFIRMHF & 50.6992 & 15.8189 & 0.0063 & 0.1911 & 53.8608 & 14.1509 & 0.0056 & 0.1489 \\
\hline
\end{tabular}

Table 9. Performance results in the image "Lena".

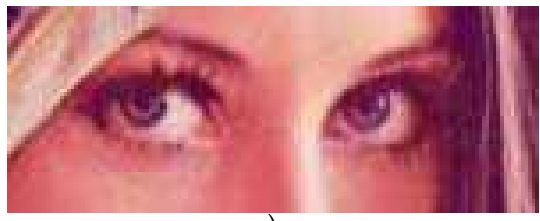

a)

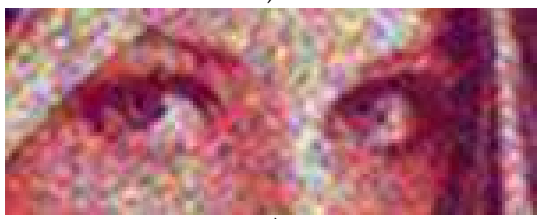

c)

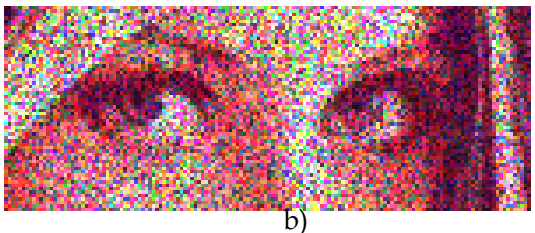

b)

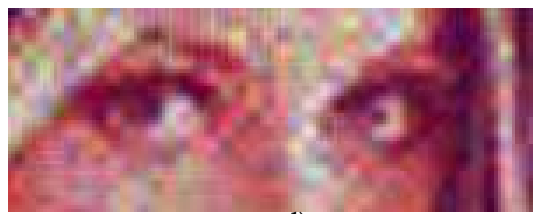

d)

Fig. 11. Subjective visual quantities of restored zoom part of color image "Lena", a) Original image, b) Input noisy image corrupted by 0.2 of variance of speckle noise in each a channel, c) WRAF filtered image; d) WMMKNNF filtered image.

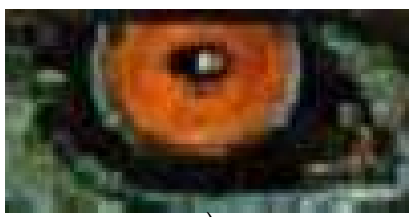

a)

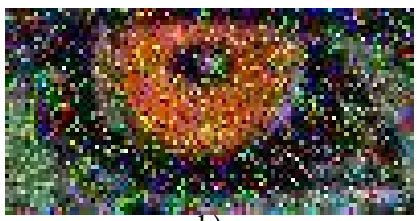

b)

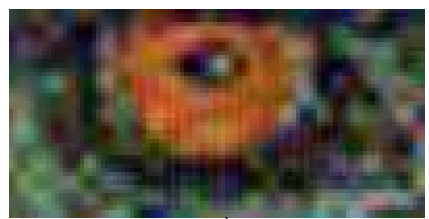

C)

Fig. 12. Subjective visual quantities of restored zoom part of the color image "Mandrill", a) Original image, b) Input noisy image corrupted by $20 \%$ impulsive noise in each a channel, c) WMMKNNF filtered image. 


\subsection{Noise suppression in 3D gray scale video sequences}

In this section, we propose $3 \mathrm{D}$ filtering algorithms to process ultrasound sequences contaminated by speckle and impulsive noise. The 3D MMKNN and 3D MML filters have been evaluated, and their performance has been compared with different nonlinear 2D filters which were adapted to 3D. The filters used as comparative ones were the modified aTrimmed Mean (MATM), Ranked-Order (RO), Multistage Median (MSM1 to MSM6), Comparison and Selection (CS), MaxMed, Selection Average (SelAve), Selection Median (SelMed), and Lower-Upper-Middle (LUM, LUM Sharp, and LUM Smooth) (Astola \& Kuosmanen, 1997) filters. These filters were computed according with their references and were adapted to 3D imaging.

An ultrasound sequence of $640 \times 480$ pixels with 90 frames (3D image of 640x480x90 voxels) was degraded with 0.05 and 0.1 of variance of speckle noise added to the natural speckle noise of the sequence. The performance results are depicted in Table 10 by use a frame of the sequence. From this Table one can see that the 3D MML filters provide the best results in comparison to other filters proposed as comparative. Figure 13 exhibits the visual results of restored images obtained by the use of different filters according to Table 10. In the Figure we observe that the proposed filters provide the better results in speckle noise suppression and detail preservation in comparison with other filters.

\begin{tabular}{|c|c|c|c|c|}
\hline \multirow{3}{*}{ 3D Filters } & \multicolumn{4}{|c|}{ Speckle noise variance } \\
\hline & \multicolumn{2}{|c|}{0.05} & \multicolumn{2}{|c|}{0.1} \\
\hline & PSNR & MAE & PSNR & MAE \\
\hline CS & 15.435 & 32.875 & 13.843 & 39.778 \\
\hline LUM Smooth & 17.915 & 25.142 & 15.440 & 33.823 \\
\hline LUM Sharp & 15.625 & 30.927 & 14.444 & 36.425 \\
\hline LUM & 15.518 & 31.427 & 14.379 & 36.748 \\
\hline MaxMed & 18.562 & 24.206 & 15.919 & 32.913 \\
\hline MATM & 20.418 & 15.124 & 19.095 & 18.663 \\
\hline MSM1 & 20.568 & 17.624 & 18.061 & 23.684 \\
\hline MSM2 & 20.484 & 17.789 & 18.038 & 23.725 \\
\hline MSM3 & 22.421 & 14.206 & 20.261 & 18.456 \\
\hline MSM4 & 21.697 & 15.401 & 19.348 & 20.351 \\
\hline MSM5 & 19.554 & 20.207 & 16.964 & 27.444 \\
\hline MSM6 & 22.083 & 14.688 & 19.744 & 19.374 \\
\hline SelAve & 21.182 & 17.647 & 19.192 & 22.814 \\
\hline SelMed & 20.836 & 15.750 & 19.013 & 20.094 \\
\hline $\mathrm{RO}$ & 21.587 & 14.520 & 19.802 & 18.179 \\
\hline MMKNN (S) & 21.554 & 15.199 & 18.949 & 20.995 \\
\hline MMKNN (H) & 21.572 & 15.169 & 19.040 & 20.798 \\
\hline MMKNN (A) & 21.399 & 14.614 & 18.640 & 20.226 \\
\hline MMKNN (B) & 22.658 & 13.309 & 20.075 & 17.819 \\
\hline MMKNN (T) & 22.499 & 13.446 & 19.855 & 18.125 \\
\hline MML (T, U,D) & 29.876 & 5.016 & 28.6175 & 5.7429 \\
\hline MML (T, L,D) & 28.797 & 5.646 & 28.188 & 6.0194 \\
\hline
\end{tabular}

Table 10. Performance results of different filters in a frame of ultrasound sequence degraded with speckle noise. 


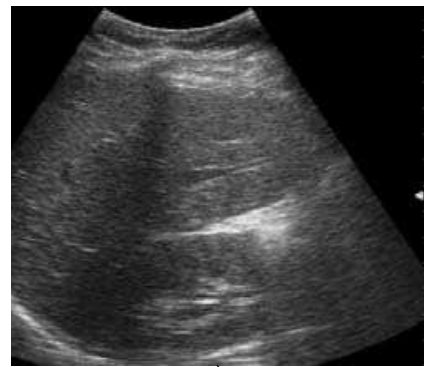

a)

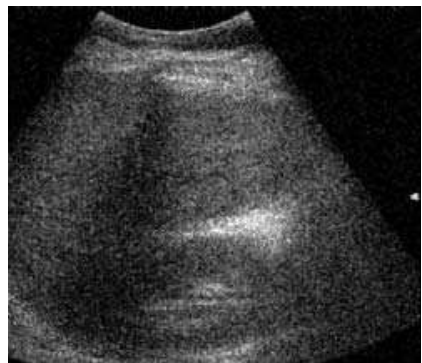

d)

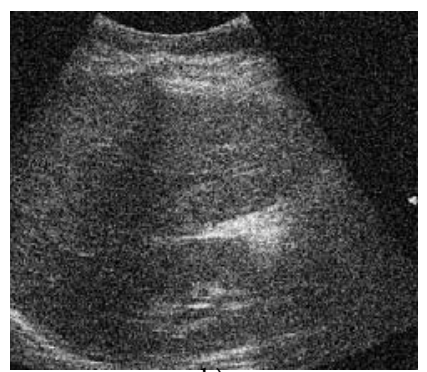

b)

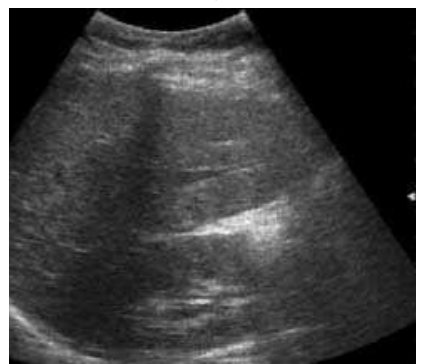

e)

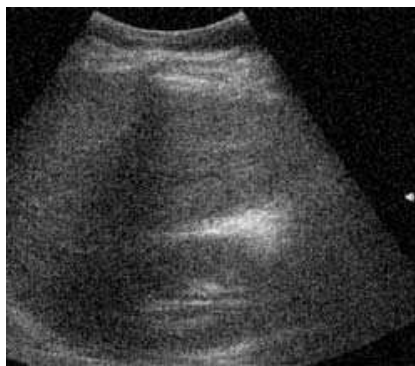

c)

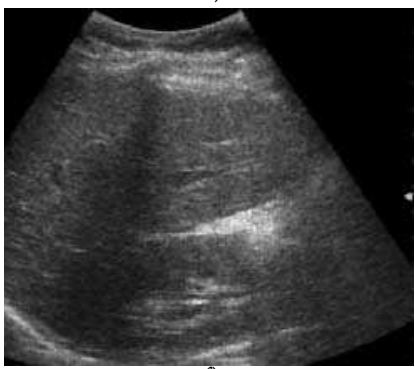

f)

Fig. 13. Visual results in a frame of ultrasound sequence. a) original frame, b) frame degraded by 0.05 of variance of speckle noise, c) restored frame by MSM6 filter, d) restored frame by MMKNN (B) filter, e) restored frame by MML filter (T,U,D), f) restored frame by MML filter (T,L,D).

\subsection{Optimal values of parameters of proposed filters}

The values of parameters for the RMKNN and MML filters and influence functions were found after numerous simulations with different images and video sequences degraded with different percentages of impulsive noise and variances of speckle noise:

a. Gray scale images and video sequences: The optimal parameters of RMKNN and MML filters are: $a=4, K_{\min }=5$, and $K_{\max }=8$; and $s=3$ and $U=15$, respectively. The values for influence functions are: $r=25, a=40$, and $\beta=200$ for Hampel, $r=35$ for Andrew, $r=15$ Tukey, and $r=20$ for Bernoulli. Therefore, the WDMML and WMMKNNF filters use the values proposed above.

b. Color images and video sequences: The values of parameters of proposed VRMKNN filters were $0.5<a<12, K_{\min }=5$, and $K_{\max }=8$, and the parameters of the influence functions were: $r \leq 81$ for Andrew, and $\alpha=10, \beta \leq 90$, and $r=300$ for Hampel.

The processing time performance of the proposed filters depends on the image to process and almost does not vary for different noise levels; these values also depend on the complex calculation of the influence functions and parameters of the proposed filter. The processing time can change with other values for these parameters, increasing or decreasing the times but the PSNR and MAE values change within the range of $\pm 10 \%$, it is due that we fix the parameters to realize the real-time implementation of proposed filters. 


\section{Conclusions}

We present the RMKNN and MML filters in spatial and wavelet domain for impulsive and speckle noise suppression in gray scale and color imaging. Extensive simulation results with different gray scale and color images and video sequences have demonstrated that the proposed filters consistently outperform other filters by balancing the tradeoff between noise suppression, fine detail preservation, color retention, and processing time.

\section{References}

Abreu, E., Lightstone, M., Mitra, S. K. \& Arakawa, K. (1996). A new efficient approach for the removal of impulse noise from highly corrupted images. IEEE Trans. Image Process., Vol.5, No.6, 1012-1025, ISSN:1057-7149

Aizenberg, I., Astola, J., Bregin, T., Butakoff, C., Egiazarian, K., \& Paily, D. (2003). Detectors of the Impulsive Noise and new Effective Filters for the Impulsive Noise Reduction, Proc. SPIE Image Process., Algorithms and Syst. II, Vol. 5014, ISBN: 9780819448149, pp. 419-428, San Jose, Ca, USA

Astola, J. \& Kuosmanen, P. (1997). Fundamentals of Nonlinear Digital Filtering, CRC Press. ISBN:0-8493-2570-6, Boca Raton-New York, USA

Bovik, A. (2000). Handbook of Image and Video Processing, Academic Press., ISBN:0121197921, San Diego CA

Chen, T., Ma, K. \& Chen, L. (1999). Tri-State Median filter for image denoising. IEEE Trans. Image Process., Vol.8, No.12, 1834-1838, ISSN:1057-7149

Chen, T. \& Wu, H. R. (2001). Adaptive impulse detection using center-weighted median filters. IEEE Signal Processing Letters, Vol.8, No.1, 1-3, ISSN:1070-9908

Gallegos-Funes, F. J., Ponomaryov, V., Sadovnychiy, S., \& Nino-de-Rivera, L. (2002). Median M-type K-nearest neighbour (MMKNN) filter to remove impulse noise from corrupted images. Electron. Lett., Vol.38, No.15, 786-787, ISSN:0013-5194

Gallegos, F., \& Ponomaryov, V. (2004). Real-time image filtering scheme based on robust estimators in presence of impulsive noise. Real Time Imaging, Vol.8, No.2, 78-90, ISSN:1077-2014

Gallegos-Funes, F., Ponomaryov, V., \& De-La-Rosa J. (2005). ABST M-type K-nearest neighbor (ABSTM-KNN) for image denoising. IEICE Trans. Funds. Electronics Comms. Computer Science, Vol.E88A, No.3, 798-799, ISSN:0916-8508

Gallegos-Funes, F., Varela-Benitez, J., \& Ponomaryov, V. (2008). Rank M-Type L (RM L)Filter for Image Denoising, IEICE Trans. Funds. Electronics, Comms. Computer Sciences, Vol.E91A, No.12, 3817-3819, ISSN:0916-8508

Gallegos-Funes, F. J., Martínez-Valdes, J., \& De-la-Rosa-Vázquez, J. M. (2007). Order statistics filters in wavelet domain for color image processing, Proc. IEEE Sixth Mexican Int. Conference on Artificial Intelligence, ISBN:978-0-7695-3124-3, pp.121-130, Mexico

Huber, P.J. (1981). Robust Statistics, Wiley, ISBN:0-471-65072-2, New York, USA

Hampel, F. R., Ronchetti, E. M., Rouseew, P. J. \& Stahel, W. A. (1986). Robust Statistics. The approach based on influence function. Wiley ISBN:0-471-73577-9, New York, USA

Kehtarnavaz, N., \& Keramat, M. (2001). DSP System Design using the TMS320C6000, Prentice Hall, ISBN:0-13-091031-7, Upper Saddle River, NJ, USA. 
Kotropoulos, C., \& Pitas, I. (1996). Adaptive LMS L-filters for noise suppression in images. IEEE Trans. Image Process., Vol.5, No.12, 1596-1609, ISSN:1057-7149

Kotropoulos, C. \& Pitas, I. (2001). Nonlinear Model-Based Image/Video Processing and Analysis, John Wiley \& Sons, ISBN:0-471-37735-X, New York

Lukin, V., Melnik, V., Chemerovsky, V., Astola, J., Saarinen, K. (1998). Hard-switching adaptive filter for speckle image processing, Proc. SPIE Mathematical Modeling and Estimation Techniques in Computer Vision, Vol. 3457, pp. 31-42, San Diego, USA

Mahbubur Rahman, S. M., \& Kamrul Hasan, Md. (2003). Wavelet-domain iterative center weighted median filter for image denoising. Signal Processing, Vol.83, No.5, 10011012, ISSN:0165-1684

Nikolaidis, N., \& Pitas, I. (2001). 3-D Image Processing Algorithms, John Wiley \& Sons, ISBN:0471-37736-8, New York, USA

Öten, R., De Figueiredo, R.J.P. (2002). Sampled-Function Weighted Order Filters. IEEE Trans. Circuits and Systems-II, Vol.49, No.1, 1-10, ISSN:1057-7130

Özkan, M. K., Sezan, M. I. \& Murat, A. (1993). Adaptive motion-compensated filtering of noisy image sequences. IEEE Trans. Circuits and Syst. for Video Tech., Vol.3, No.4, 277-290, ISSN:1051-8215

Peltonen, S. \& Kuosmanen, P. (2001). Robustness of nonlinear filters for image processing. J. Electronic Imaging, Vol.10, No.3, 744-756, ISSN:1560-229X

Plataniotis, K. N., Androutsos, D., Vinayagamoorthy, S. \& Venetsanopoulos, A. N. (1997). Color image processing using adaptive multichannel filters. IEEE Trans. Image Process., Vol.6, No.7, 933-949,. ISSN:1057-7149

Plataniotis, K. N., \& Venetsanopoulos, A. N. (2000). Color Image Processing and Applications, Springer-Verlag, Berlin Heidelberg, ISBN:3-540-66953-1

Ponomaryov, V., Gallegos-Funes, F., Rosales-Silva, A. (2005). Real-Time Color Image Processing Using Order Statistics Filters. Journal of Mathematical Imaging and Vision, Vol. 23, No. 3, 315-319, ISSN:0924-9907

Texas Instruments (1998). TMS320C62x/67x Programmer's Guide, SPRU198D, Texas Instruments Incorporated. Dallas, USA

Trahanias, P. E., Karakos, D. G., \& Venetsanopoulos, A. N. (1996). Directional processing of color images: Theory and experimental results. IEEE Trans. Image Process., Vol.5, No.6, 868-880, ISSN:1057-7149

Varela-Benítez, J. L., Gallegos-Funes, F. J., \& Ponomaryov, V. I. (2007a). Real-time speckle and impulsive noise suppression in 3-D imaging based on robust linear combinations of order statistics, Proc. SPIE Real-Time Image Processing 2007, Vol.6496, ISBN:9780819466099, pp. 64960H, San Jose, USA

Varela-Benitez, J. L., Gallegos-Funes, F., Ponomaryov, V., De la Rosa Vazquez, J. M. (2007b). RM L-filters in wavelet domain for image processing applications, Proc. IEEE Sixth Mexican Int. Conference on Artificial Intelligence, ISBN:978-0-7695-3124-3, pp.113-120, Mexico.

Walker, J. S. (1999). A Primer on Wavelets and their Scientific Applications, Chapman \& Hall/CRC, ISSN:0-8493-8276-9, Boca Raton-New York, USA

Wei-Yu, H. \& Ja-Chen, L. (1997). Minimum-maximum exclusive mean (MMEM) filter to remove impulse noise from highly corrupted images. Electronics Letters, Vol.33, No.2, 124-125, ISSN:0013-5194 


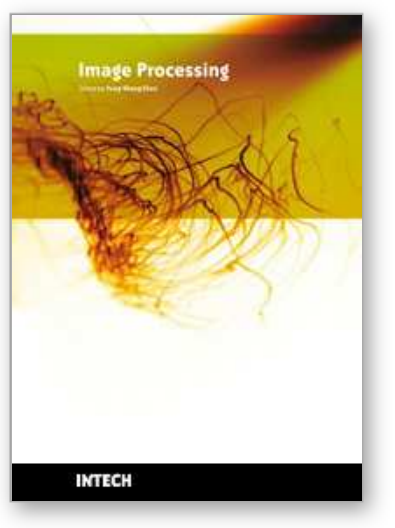

\author{
Image Processing \\ Edited by Yung-Sheng Chen
}

ISBN 978-953-307-026-1

Hard cover, 516 pages

Publisher InTech

Published online 01, December, 2009

Published in print edition December, 2009

There are six sections in this book. The first section presents basic image processing techniques, such as image acquisition, storage, retrieval, transformation, filtering, and parallel computing. Then, some applications, such as road sign recognition, air quality monitoring, remote sensed image analysis, and diagnosis of industrial parts are considered. Subsequently, the application of image processing for the special eye examination and a newly three-dimensional digital camera are introduced. On the other hand, the section of medical imaging will show the applications of nuclear imaging, ultrasound imaging, and biology. The section of neural fuzzy presents the topics of image recognition, self-learning, image restoration, as well as evolutionary. The final section will show how to implement the hardware design based on the SoC or FPGA to accelerate image processing.

\title{
How to reference
}

In order to correctly reference this scholarly work, feel free to copy and paste the following:

Francisco J. Gallegos-Funes and Alberto J. Rosales-Silva (2009). Rank M-type Filters for Image Denoising, Image Processing, Yung-Sheng Chen (Ed.), ISBN: 978-953-307-026-1, InTech, Available from: http://www.intechopen.com/books/image-processing/rank-m-type-filters-for-image-denoising

\section{INTECH}

open science | open minds

\section{InTech Europe}

University Campus STeP Ri Slavka Krautzeka 83/A 51000 Rijeka, Croatia Phone: +385 (51) 770447 Fax: +385 (51) 686166 www.intechopen.com

\section{InTech China}

Unit 405, Office Block, Hotel Equatorial Shanghai No.65, Yan An Road (West), Shanghai, 200040, China 中国上海市延安西路65号上海国际贵都大饭店办公楼 405 单元 Phone: +86-21-62489820

Fax: $+86-21-62489821$ 
(C) 2009 The Author(s). Licensee IntechOpen. This chapter is distributed under the terms of the Creative Commons Attribution-NonCommercialShareAlike-3.0 License, which permits use, distribution and reproduction for non-commercial purposes, provided the original is properly cited and derivative works building on this content are distributed under the same license. 